\title{
Study of dielectric permittivity and fatty acid composition for fats and oil in wide frequency spectroscopy measurement at $0.5-50 \mathrm{GHz}$
}

\begin{abstract}
The study focused on application of spectral permittivity technique subjected to wide frequency range of from microwaves to millimeter waves (i.e. $0.5-50 \mathrm{GHz}$ ) at the temperature of $60^{\circ} \mathrm{C}$ to understand the relationship of dielectric permittivity and fatty acid composition in fats and oil. Results showed that the dielectric constant of 4 animal fats and 2 vegetable oil exhibited same frequency dependence. The dielectric constant of fats and oil increases with an increase in the degree of unsaturation in vegetable oil at 7.4, 13.4, 18.3, 23.3, and 46.0 $\mathrm{GHz}$. The dielectric constant decreases with the increase of composition of palmitic and oleic acid in animal fat at 13.4 and $18.3 \mathrm{GHz}$ respectively. The understanding obtained in this work could be useful towards distinguishing fats and oils especially lard using rapid permittivity spectroscopy based on accurate chemical composition, suitable for Halal product analysis.
\end{abstract}

Keyword: Animal fats; Fatty acid methyl ester (FAME); Spectral permittivity; Vegetable oil 